\section{Cancer et développement}

Les relations entre cancer et développement sont connues depuis longtemps, illustrées par le concept des marqueurs oncofotaux". La prolifération cellulaire est intense au cours du développement, et la différenciation est perturbée au cours du cancer: Aussi, ne faut-il pas s'étonner du nombre croissant de gènes du développment, souvent caractérisés chez la drosophile ou un autre organisme modèle, qui se révèlent à l'origine de cancers chez l'homme. Ce mois-ci, le système de transmission $d u$ signal Hedgehog est à l'honneur. Son régulateur négatif, Patched, est déficient dans le syndrome de Gorlin et des épithéliomas basocellulaires sporadiques. L'une des cibles du signal Hedgehog est le facteur Wingless/Wnt1, lui-même impliqué dans la régulation de la prolifération et de la morphogenèse. Ce facteur agit en contrôlant la quantité et l'activité de la $\beta$ caténine (et de son homologue Armadillo chez Drosophila melanogaster), peut-être par l'intermédiaire d'APC, produit du gène de la polypose colique familiale, impliqué aussi dans la majorité des cancers coliques sporadiques. Certaines protéines Hedgehog sont également essentielles à la différenciation cartilagineuse et au développement de l'os, et sont certainement candidates à jouer un rôle dans les tumeurs de ces tissus.

\title{
Naevomatose baso-cellulaire et gène patched: un nouveau lien entre cancer et gènes du développement
}

Une nouvelle fois, la génétique du développement vient à la rencontre de la génétique humaine avec la publication par deux équipes dans Cell et Science, de l'identification du gène du syndrome de Gorlin (ou nævomatose baso-cellulaire), homologue humain du gène de drosophile patched.

La naevomatose baso-cellulaire (NBC) ou syndrome de Gorlin [1] est une maladie génétique autosomique dominante caractérisée par un spectre d'anomalies du développement et une prédisposition à différents cancers. Il s'agit d'une maladie bien individualisée avec plus de 600 cas rapportés. La NBC associe principalement des naevus baso-cellulaires, une porokératose palmoplantaire*, des kystes épidermoïdes des maxillaires, des anomalies osseuses costo-vertébrales, des calcifications ectopiques intracrâniennes (faux du cerveau, tente de l'hypophyse), une dysmorphie faciale (hypertélorisme**) avec macrocéphalie [2], à diverses manifestations neurologiques, oculaires, génitales ou endocriniennes [3]. Chez les jeunes enfants, la recherche d'une calcification ectopique sur la radiographie simple du crâne est un excellent critère, car il est précoce, caractéristique dans un contexte familial, et très fréquent (environ $90 \%$ des cas). Le potentiel carcinologique fait la gravité de cette maladie, justifiant un dépistage précoce

\footnotetext{
* Kérntose ponctuée carractérisée par de petits éléments cornés, durs, transhucides, enchâssés dans l'épiderme plantaire et palmaire et creusés de petites cavités (pits).

** Elargissement anormal de l'espace interoculaire.
}

et une surveillance régulière et prolongée des patients et de leur descendance. Le cancer le plus fréquemment retrouvé dans le syndrome est le carcinome baso-cellulaire qui est le plus commun des cancers chez l'homme. D'autres tumeurs fréquentes sont des fibromes ovariens et des médulloblastomes [4]. Il s'agit d'une maladie à pénétrance complète et à expressivité variable ce qui peut poser des problèmes de diagnostic. Certains auteurs font de la NBC la cinquième phacomatose ${ }^{* * *}$. Les naevus baso-cellulaires se développent à partir de l'ébauche épithéliale primitive et les kystes maxillaires des restes épithéliaux odontogènes. La description d'anomalies du système nerveux renforce l'intégration de ce syndrome dans le cadre des neuroectodermoses: phacomatoses (ou hamartoses) ou neurocristopathies****. Des cellules de la crête neurale dérivent les mélanoblastes et le mésectoderme qui donne l'essentiel du mésenchyme du premier arc branchial et de son dérivé, le maxillaire inférieur ainsi que les odontoblastes. Cet ectomésenchyme principal participe à la genèse du squelette céphalique, ce qui pourrait expliquer la dysmorphie craniofaciale, les kystes mandibulaires et les calcifications méningées, puisque pie-mère

*** Affection congénitale comportant des tumeurs de petite taille (phacomes) d'origine ectodermique. **** (On réunit traditionnellement sous le nom de neurocristopathies des affections dysplasiques touchant des dérivés de la crête neurale. En fait, l'exemple du syndrome de Corlin et du rôle de l'atched montrent que cette appellation peut n'avoir aucune base physiopathologique. Jans le cas présent, par exemple, les tissus cibles du déficit en Patched ne sont pas du tout limités aux dérivés de la crête neurale. 
et arachnoïde dérivent du même mésenchyme. Le déterminisme malformatif costo-vertébral pourrait avoir une origine mixte mésodermique et ectomésodermique, en provenance des cellules issues des crêtes neurales situées au niveau des premiers somites, au moins dans la région cervico-dorsale [5].

Le gène de la NBC a été localisé dans l'intervalle 9q22.3-q31 par des études familiales de liaison génétique [6]. L'étude de tumeurs dans la NBC avec des marqueurs du chromosome $9 \mathrm{q}$ a montré une perte de l'hétérozygotie pour cette région [7], suggérant que le gène de la NBC pourrait correspondre à un gène suppresseur de tumeur [8].

L'état d'haplo-insuffisance du Gorlin, et la date variable de survenue de la perte d'hétérozygotie du second allèle, laissent supposer trois types de mécanismes moléculaires à l'origine des différentes lésions anatomopathologiques: premièrement, les malformations symétriques présentes chez tous les patients, telles que la macrocéphalie et la dysmorphie faciale, doivent résulter d'un trouble quantitatif de la voie de transduction passant par la protéine Patched (voir ci-dessous); deuxièmement, les malformations sporadiques asymétriques, généralement groupées, présentes chez un même patient, telles que les malformations costo-vertébrales, résultent probablement d'une perte allélique ou d'une inactivation précoce de l'allèle normal dans les cellules souches d'un tissu donné a u cours du développement; enfin, le troisième mécanisme correspond à la perte de l'allèle normal au cours de la vie adulte et conduit à des cancers basocellulaires multisites caractéristiques. Lors du quatrième atelier sur le chromosome $\mathrm{n}^{\circ} 9$, au printemps 1995 , le gène du syndrome de Gorlin était positionné en 9q22 entre les marqueurs D9S287 et 196. Hahn et al. $[9,10]$ utilisant la stratégie classique du clonage positionnel identifièrent finalement un gène $P T C$ contenant 22 exons et présentant $67 \%$ d'identité au niveau de l'ADN avec le gène patched (ptc) de drosophile. Les auteurs décrivent six mutations non-sens présentes chez

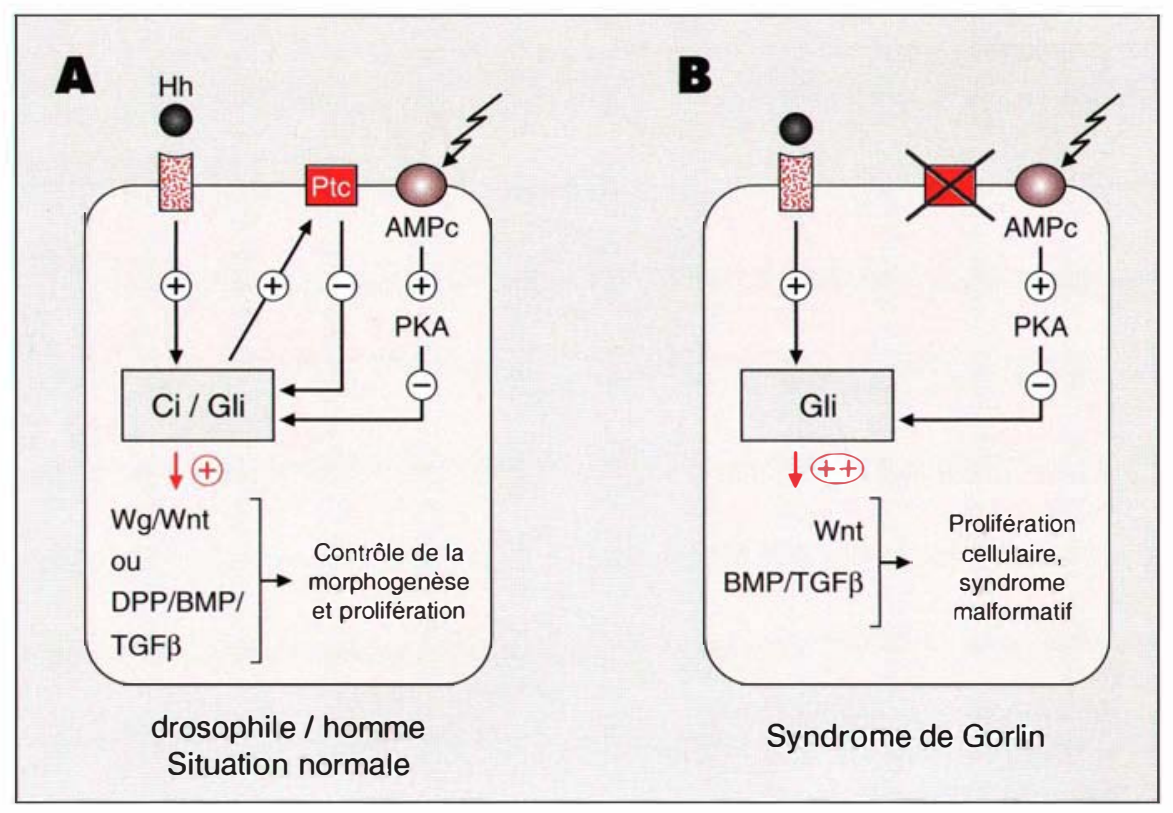

Figure 1. Schéma de la voie de transduction Hedgehog/Patched. A. Situation normale : La signalisation cellulaire par un facteur sécrété de la famille Hedgehog $(\mathrm{Hh})$, probablement relayé par un récepteur à sept passages transmembranaires dénommé Smoothened [20], active la transcription d'une batterie de gènes cibles, comprenant toujours un second facteur sécrété lde type Wnt ou Decapentaplegic/BMP (bone morphogenic protein)/TGF $\beta$ ) et de celle du gène patched PTC, et contrôle ainsi la morphogenèse ou la prolifération cellulaire [15-18]. Cette activation par un facteur de type Hedgehog s'oppose à une inhibition constitutive par la PKA et la protéine Patched. Un facteur de transcription (Ci, cubitus interruptus, chez la drosophile, ou de la famille Gli chez les vertébrés) pourrait être le site où sont intégrés les différents tonus, positif imprimé par Hedgehog et négatifs imprimés par la PKA et Patched [18, 19]. On peut supposer que l'activité de la PKA, dépendante de I'AMP cyclique, est, elle aussi, soumise à la régulation de signaux extracellulaires empruntant la voie de récepteurs non encore identifiés. L'activation de la transcription de patched agit donc comme un rétrocontrôle négatif dans ce dispositif de signalisation cellulaire [15, 18]. B. Chez les malades atteints du syndrome de Gorlin, le rétrocontrôle négatif passant par Patched est perdu, ce qui aboutit à une dépression constitutive de l'expression des facteurs Wnt, TGF $\beta, B M P$, etc. On ne sait pas encore si, dans ce cas, une hyperstimulation de la seconde voie de contrôle négatif passant par l'AMP cyclique peut prendre le relais de Patched.

novo, non observée chez les parents), ainsi que deux autres mutations accompagnées d'une perte allélique de la région critique chez deux patients atteints de la forme sporadique du carcinome baso-cellulaire. Ces mutations sont de type transition, et délétion ou insertion de quelques paires de bases, et ont été détectées par SSCP et séquençage. Dans un des cas sporadiques, une mutation de CC $\rightarrow$ TT est compatible avec l'effet mutagène des UVB, ce qui est en accord avec le rôle de l'exposition au soleil dans l'apparition de ces cancers $[9,11]$.
Dans une étude séparée, Johnson et al. [11] isolèrent le gène $P T C$ humain après avoir isolé le gène $p t c$ chez la souris; ils localisèrent l'homologue humain dans la région du Gorlin en 9q22. Ils identifièrent alors une insertion de 9 paires de bases et une délétion de 11 pb dans deux familles distinctes atteintes du syndrome de Gorlin, et une mutation $\mathrm{C} \rightarrow \mathrm{T}$ chez un patient atteint d'un carcinome baso-cellulaire sporadique, à nouveau compatible avec l'effet mutagène des UVB.

Patched a été initialement identifié chez la drosophile; il code pour une 


\begin{tabular}{|c|c|}
\hline \multicolumn{2}{|c|}{ Tableau I } \\
\hline \multicolumn{2}{|c|}{ SITE D'EXPRESSION DE PATCHED AU COURS DU DÉVELOPPEMENT } \\
ET MANIFESTATIONS DU SYNDROME DE GORLIN
\end{tabular}

glycoprotéine transmembranaire comportant 12 domaines et pourrait fonctionner comme récepteur ou transporteur. Ptc, chez la drosophile, inhibe le signal apporté par hedgehog $(h h)$ et donc l'induction de différents gènes cibles impliqués dans la communication intercellulaire, comme decapentaplegic $(d p p)$ et ptc luimême $[12,13]$. Les homologues de Ptc chez la souris, le poulet et le poisson-zèbre ont été récemment clonés [14-16]. L'étude du profil d'expression de Ptc au cours de l'embryogenèse chez les vertébrés a permis d'associer l'ensemble des manifestations cutanées et extracutanées de la NBC à une expression spécifique de $P T C$ au cours du développement et de la différenciation des tissus (Tableau I) [9, 14-16]. Chez la drosophile, la voie de transduction Hedgehog/Patched est impliquée à de multiples reprises au cours du développement (figure 1). De très nombreux travaux ont récemment démontré que cette voie de signalisation est largement conservée dans le règne animal $[12-14,16-17]$. Elle participe, entre autres, au cours du développement des vertébrés, à l'organisation de la plaque neurale (différenciation des cellules du plancher et des neurones moteurs par la chorde), ainsi qu'à la détermination de l'axe antéro-postérieur du membre $[12,13]$. Ces derniers auteurs spéculent que la perte de fonction de Ptcchez les vertébrés doit conduire à une expression ectopique des gènes cibles de Hedgehog, codant, en particulier, pour un second facteur de croissance de type Wnt ou BMP/TGF $\beta$. En effet, l'activation de la voie Hedgehog, peut-être relayée par un facteur à doigt de zinc de type Gli, aboutit normalement aussi à l'augmentation de la synthèse du régulateur négatif Patched qui rétrocontrôle donc le système. C'est ce rétrocontrôle qui serait perdu en cas de mutation inactivatrice du gène $P T C$. Le rôle de $P T C$ comme gène suppresseur de tumeur révèle donc de nouveaux rôles pour Hedgehog, et suggère que ce dernier est impliqué dans la prolifération cellulaire de la peau chez l'adulte. Trois gènes Hedgehog fortement conservés ont été identifiés chez les vertébrés (sonic, desert et indian). Ainsi, il serait intéressant d'identifier lequel des trois est exprimé dans la peau.

Au contraire de $\mathrm{Rb}$ ou $\mathrm{p} 53$, protéines suppresseurs de tumeurs nucléaires interagissant avec des composants de la machinerie du cycle cellulaire [8], PTC est une protéine membranaire contrôlant une répression transcriptionnelle. $P T C$ représente le premier gène de développement qui soit aussi un gène suppresseur de tumeur. En effet, seuls quelques gènes du développement ont été identifiés à ce jour comme oncogènes tels que WNT1 (homologue du gène wingless) et GLI (gène du glioblastome humain et homologue du gène cubitus interruptus de drosophile). Or, ces deux gènes apparaissent comme des cibles directes de la répression exercée par
PTC en présence de Hedgehog éclairant de fait la nature antioncogénique de $P T C$ [13].

Bien que d'évolution lente et de malignité locale (les métastases étant exceptionnelles), l'épithélioma basocellulaire représente un problème important en dermato-cancérologie puisque plus de 750000 cas ont été enregistrés aux États-Unis en 1995. Il sera ainsi important d'évaluer, en dehors de la NBC, l'état d'homozygotie nulle pour le gène $P T C$ dans les formes sporadiques de ces cancers baso-cellulaires. Cet intérêt va plus loin, avec la possibilité de développement de nouvelles approches thérapeutiques fondées sur notre connaissance de la voie de transduction Hedgehog/PTC. En effet, la présence d'une deuxième voie d'inhibition du signal Hedgehog a été mise en évidence chez la drosophile qui fait intervenir la protéine kinase dépendante de l'AMPc (PKA) dont les agonistes biodisponibles pourraient ainsi représenter une cible thérapeutique éventuelle [13].

Cet espoir thérapeutique pourrait bénéficier aux patients atteints du syndrome de Gorlin. Toutefois, une des premières tâches de la communauté médico-scientifique sera d'évaluer la corrélation phénotype/génotype de la NBC en identifiant les diffférentes mutations du gène $P T C$ présentes dans ce syndrome

\section{TIRÉS À PART}

P. Gorry.

\section{RÉFÉRENCES}

1. Gorlin RJ, Goltz RW. Multiple nevoid basal cell epithelioma, jaw cysts and bifid rib: a syndrome. N Engl J Med 1960; 262: 908-12.

2. Soekarman D, Fryns JP, Casaer P, Van den Berghe $H$. Increased head circumference and facial cleft as presenting signs of nevoid basal-cell carcinoma syndrome. Genet Counsel 1991 ; 2 : 157-62.

3. Shanley S, Ratcliffe J, Hockey, A Haan E, Oley C, Ravine D, Martin N, Wicking C, Chenevix-Trench $G$. Nevoid basal cell carcinoma syndrome: review of 118 affected individuals. Am J Med Genet 1994; 50 : 282-90.

4. Lacombe D, Chateil JF, Fontan D, Battin J. Medulloblastoma in the nevoid basal cell carcinoma syndrome : case reports and review of the litterature. Genet Counsel 1990; 1: 273-7. 


\section{RÉFÉRENCES}

5. Fauchet R. Le syndrome de Gorlin ou naevomatose baso-cellulaire. Manifestations squelettiques et hypothèse pathogénique des déterminations costo-vertébrales. Ann Med Phys 1981; 24 : 28-40.

6. Farndon PA, Del Mastro RG, Evans DGR, Kilpatrick MW. Location of gene for Gorlin Syndrome. Lancet $1992 ; 339: 581-2$.

7. Gailani MR, Bale SJ, Leffell DJ, et al. Development defects in Gorlin syndrome related to a putative tumor suppressor gene on chromosome 9. Cell $1992 ; 69$ : 111-7.

8. Thomas G. Dix ans de recherche sur les prédispositions génétiques au développement des tumeurs. médecine/sciences $1995 ; 11$ : $336-48$.

9. Hahn H, Wicking C, Zaphiropoulos PG, et al. Mutations of the human homolog of drosophila patched in the nevoid basal cell carcinoma syndrome. Cell 1996 ; 85 : 841-51.

10. Hahn H, Christiansen J, Wicking C, Zaphiropoulos PG, Shanley S, Chidambaram A, Gerrard B, Vorechovsky I, Bale AE, Toftgard AM, Dean M, Wainwright B. A mammalian patched homolog is expressed in target tissues of sonic hedgehog and maps to a region associated with developmental abnormalities. J Biol Chem 1996 ; 271 : 12125-8.

11. Johnson RL, Rothman AL, Xie J, Goodrich LV, Bare JW, Bonifas JM, Quinn AG,
Myers RM, Cox DR, Epstein EH, Scott MP. Human homolog of patched, a candidate gene for the basal cell nevus syndrome. Science $1996 ; 272$ : 1668-71.

12. Concordet J. Morphogenèse, acide rétinoîque et Sonic Hedgehog. médecine/sciences $1994 ; 10: 570-3$

13. Perrimon N. Hedgehog and beyond. Cell $1995 ; 80: 517-20$

14. Goodrich LV, Johnson RL, Milenkovic L, McMahon JA, Scott MP. Conservation of the hedgehog/patched signaling pathway from flies to mice: induction of a mouse patched gene by Hedgehog. Genes Dev 1996; 10: $301-12$.

15. Marigo V, Scott MP, Johnson RL, Goodrich LV, Tabin CJ. Conservation in Hedgehog signaling: induction of a chicken patched homolog by Sonic hedgehog in the developing limb. Development 1996; 122: 1225-33.

16. Concordet JP, Lewis KE, Moore JW, Goodrich LV, Johnson RL, Scott MP Ingham PW. Spatial regulation of a zebrafish patched homolog reflects the roles of somic hedgehog and protein kinase $\mathrm{A}$ in neural tube and somite patterning. Development 1996 ; 122 : 2847-57.

17. Fietz MJ, Concordet JP, Barbosa R, Johnson R, Krauss S, McMahon AP, Tabin $\mathrm{C}$, Ingham PW. The hedgehog gene family in Drosophila and vertebrate development. Development 1994 ; 36 (suppl) : 43-51.
18. Forbes AJ, Nakano Y, Taylor AM, Ingham PW. Genetic analysis of hedgehog signalling in the Drosophila embryo. Development 1993 ; 29 (suppl) : 115-24.

19. Dominguez M, Brunner M, Hafen E, Basler $\mathrm{K}$. Sending and receiving the Hedgehog signal : control by the Drosophila Gli protein cubitus interruptus. Science 1996; 272 : 1621-5. 20. Van den Heuvel M, Ingham PW. Smoothened encodes a receptor-like serpentine protein required for hedgehog signalling. Nature 1996; 382 : 547-51.

\section{Philippe Gorry}

Laboratoire de Biologie Cellulaire, Université de Bordeaux II, 33076 Bordeaux Cedex.

\section{Didier Lacombe}

Service de Pédiatrie et Génétique Médicale, Hôpital Pellegrin-Enfants, Université de Bordeaux II, place Amélie-Raba-Léon, 33076 Bordeaux Cedex.

Réseau clinique Inserm SC11 "Narvomatose baso-cellulaire (syndrome de Gorlin)».

\section{Jean-Paul Concordet}

Inserm U. 129, CHU Cochin, 24, rue du Faubourg-Saint-Jacques, 75014 Paris. 